\title{
Multidrug-resistant, Extensively drug-resistant and Pandrug-resistant bacteria and antimicrobial therapy in combination
}

\author{
${ }^{1}$ S.M Shamsuzzaman \\ ${ }^{1}$ Department of Microbiology, Dhaka Medical College, Dhaka.
}

Key wards: Antibiotic combination, Bacteria, MDR, PDR, Treatment, XDR.

Frequently we are facing bacteria which are resistant to most and in some cases all available antibiotics and we call them multidrug-resistant (MDR), extensively drug-resistant (XDR) and sometimes pandrug-resistant (PDR) bacteria ${ }^{1,2,3}$. Many different definitions of MDR, XDR and PDR bacteria are being used to characterize the different patterns of resistance 1,4 . To overcome this problem. a joint initiative was taken by the European Centre for Disease Prevention and Control (ECDC) and the Centers for Disease Control and Prevention (CDC), to standardize definitions of MDR, XDR and PDR to describe acquired resistance profiles in bacteria responsible for healthcare-associated infections, such as., Staphylococcus aureus, Enterococcus spp., Enterobacteriaceae (other than Salmonella and Shigella), Pseudomonas aeruginosa and Acinetobacter spp ${ }^{5}$.

Accordingly, MDR was defined as acquired nonsusceptibility to at least one agent in three or more antimicrobial categories, XDR was defined as nonsusceptibility to at least one agent in all but two or fewer antimicrobial categories (i.e. bacterial isolates remain susceptible to only one or two categories) and PDR was defined as non-susceptibility to all agents in all antimicrobial categories $^{5}$. To ensure correct application of these definitions, bacterial isolates should be tested against all or nearly all of the antimicrobial agents within the antimicrobial categories. The major antimicrobial categories are penicillins, cephalosporins, quinolones, aminoglycosides, carbapenems, monobactams, macrolides, polymixins, glycopeptides etc.

\section{$\triangle \mathrm{C}$ Correspondence:}

Professor Dr. S.M. Shamsuzzaman

Department of Microbiology

Dhaka Medical College, Dhaka

Tel: 01819289739

Email: smszaman@yahoo.com
Initially, the term XDR was used to describe extensively drug-resistant Mycobacterium tuberculosis (XDR MTB) and was defined as 'resistance to the first-line agents isoniazid and rifampicin, to a fluoroquinolone and to at least one of the three-second-line parenteral drugs (i.e. amikacin, kanamycin or capreomycin) ${ }^{6,7}$. Two sets of criteria have mainly been used to characterize bacteria as XDR. The first is based on the number of antimicrobials or classes or subclasses to which a bacterium is resistant, and the second one whether they are 'resistant to one or more key antimicrobial agents ${ }^{8,9,10}$.

PDR : 'Pan' means 'all', pandrug resistant (PDR) means 'resistant to all antimicrobial agents'. It means that, to characterize a bacteria as PDR, it must be tested and found to be resistant to all approved and useful antimicrobials. Examples of current definitions are: 'resistant to almost all commercially available antimicrobials','resistant to all antimicrobials routinely tested'and 'resistant to all antibiotic classes available for empirical treatment' ${ }^{4,11,12}$.

How to treat these bacteria?

Some alternative drugs are available to treat MDR bacteria and very few options available to treat XDR bacteria. But almost no option is available to treat PDR bacteria. To treat PDR, either new effective antibiotics are to be discovered, alternatively best possible combinations of two or more antibiotics are to be tried. Against infections with Pseudomonas aeruginosa or Acinetobacter baumannii isolates that are resistant to all antibiotics except the polymyxins, several novel antibiotic combinations demonstrate increased activity in vitro compared with that of any single agent $3,13,14$. When two antibiotics are used in combination, the best and most desirable effect is synergism and the worst one is antagonism, summation and indifference are in between. ${ }^{15}$ In vitro synergistic effects have been observed against Pseudomonas aeruginosa ${ }^{3}$, and in vitro and in vivo in rat model synergistic effects have been observed 
against Acinetobacter baumannii using combinations of amikacin with carbapenems, and carbapenem with ceftazidyme ${ }^{14}$.

In a nosocomial outbreak of pulmonary infection with multidrug-resistant $P$. aeruginosa, cefepime and amikacin were found to be the most effective antibiotics and to be highly synergistic by checkerboard and killing curve methods ${ }^{16}$. In another cohort study, critically ill patients with respiratory tract infections due to multidrug-resistant $A$. baumannii or $P$. aeruginosa were treated with polymyxin B in combination with carbapenems were effective in $65 \%$ cases and polymixins with amikacin was effective in $28 \%$ cases ${ }^{17}$ Therefore, to treat XDR or PDR bacteria which are resistant to carbapenems and polymixins, combination of carbapenem with amikacin may be used. Alternatively carbapenem with ceftazidyme or colistin with carbapenems may also be used.

\section{References}

1. Tseng YC, Wang JT, Wu FL, Chen YC, Chie WC, Chang SC. Prognosis of adult patients with bacteremia caused by extensively resistant Acinetobacter baumannii. Diagn Microbiol Infect Dis 2007; 59: 181-190.

2. Farzana R, Shamsuzzaman S, Mamun KZ.Isolation and molecular characterization of New Delhi metallo-betalactamase-1 producing superbug in Bangladesh. J Infect Dev Ctries. 2013 Mar 14;7(3):161-8. doi: 10.3855/jidc. 2493 .

3. Farzana A. Detection of bla-NDM1 from imipenem resistant Pseudomonas aeruginosa isolated from burn unit of $\mathrm{DMCH} \&$ invitro evaluation of different antibiotic combination. Department of Microbiology, Dhaka Medical College, January 2013, [M.Phil thesis].

4. Kuo LC, Teng LJ, Yu CJ, Ho SW, Hsueh PR. Dissemination of a clone of unusual phenotype of pandrug-resistant Acinetobacter baumannii at a university hospital in Taiwan. J Clin Microbiol 2004; 42: 1759-1763.

5. Magiorakos AP, Srinivasan A, Carey RB et al. Multidrug-resistant, extensively drug-resistant and pandrug-resistant bacteria: an international expert proposal for interim standard definitions for acquired resistance. Clin Microbiol Infect Dis, 18: 268-281

6. CDC. Emergence of Mycobacterium tuberculosis with extensive resistance to second-line drugs - Worldwide, 20002004. MMWR Morb Mortal Wkly Rep 2006; 55: 301-305.

7. CDC. Notice to readers: revised definition of extensively drug-resistant tuberculosis. MMWR Morb Mortal Wkly Rep 2006; 55: 1176.
8. Cohen AL, Calfee D, Fridkin SK et al. Recommendations for metrics for multidrug-resistant organisms in healthcare settings: SHEA/ HICPAC Position paper. Infect Control Hosp Epidemiol 2008; 29: $901-913$.

9. Hidron AI, Edwards JR, Patel J et al. NHSN annual update: antimicrobial- resistant pathogens associated with healthcare-associated infections: annual summary of data reported to the National Healthcare Safety Network at the Centers for Disease Control and Prevention,

2006-2007. Infect Control Hosp Epidemiol 2008; 29: 9961011.

10.Siegel JD, Rhinehart E, Jackson M, Chiarello L. Management of multidrug-resistant organisms in health care settings, 2006. Am J Infect Control 2007; 35 (suppl 2): 165-193.

11. Falagas ME, Koletsi PK, Bliziotis IA. The diversity of definitions of multidrug-resistant (MDR) and pandrugresistant (PDR) Acinetobacter baumannii and Pseudomonas aeruginosa. J Med Microbiol 2006; 55: 1619-1629.

12. Kuo LC, Yu CJ, Lee LN et al. Clinical features of pandrug-resistant Acinetobacter baumannii bacteremia at a university hospital in Taiwan. J Formos Med Assoc 2003; 102: 601-606.

13. Rahal JJ. Novel Antibiotic Combinations against Infections with Almost Completely Resistant Pseudomonas aeruginosa and Acinetobacter Species. Clin Infect Dis 2016; 63:S95-S98.

14. Uddin BMM. In vitro and in vivo evaluation of antibiotic combination against imipenem resistant Acinetobacter baumannii isolated from patients of $\mathrm{DMCH}$. Department of Microbiology, Dhaka Medical College, January 2016 [M.Phil thesis]).

15. Brooks GF, Carroll KC, Butel JS, Morse SA, Mietzner TA. Antimicrobial chemotherapy. In: Jawtz, Melnick \& Adelberg's Medical Microbiology. USA, McGraw-Hill companies Inc. 26th edn. 2013: p 371-405.

16. Dubois V, Arpin C, Melon M, et al. Nosocomial outbreak due to a multiresistant strain of Pseudomonas aeruginosa P12: efficacy of cefepime-amikacin therapy and analysis of beta-lactam resistance. J Clin Microbiol 2001;39:2072-8.

17. Sobieszczyk ME, Furuya EY, Hay CM, et al. Combination therapy with polymyxin $\mathrm{B}$ for the treatment of multidrug-resistant gram-negative respiratory tract infections. J Antimicrob Chemother 2004;54:566-9. 